\title{
A New Approach of Image denoise Based on The Steerable Pyramid Wavelet
}

\author{
Yin Ming, Wang liwei, \\ School of Electric and Electronic Infomation, SouthWest University for Nationality \\ Chengdu, Sichuan 610000, China \\ yinming@swun.cn,wangliwei@swun.cn
}

\begin{abstract}
Keywords: non-local means; wavlet ;image denoise; Steerable Pyramid
Abstract. In this paper, we proposed a new method which exploit the generalized Gaussian distribution (GCD) as the modeling of the sub-band coefficients and estimate the parameter corresponding. We use the generalized non-local means expression and propose an generalized non-local means algorithm based on the steerable pyramid and GGD. Simulation results show the performance of the proposed methods have better visual and in terms of the peak mean squared error. Steerable Pyramid is a flexible wavelet decomposition method. The statistics of the wavelet coefficients is non-Gaussian, which follow the generalized Gaussian distribution (GGD).
\end{abstract}

\section{Introduction}

In the procedure of the imaging, it is always couple with the additional noise by the image peripheral. The noise has the probability density distribution of the Gaussian distribution. Image denoising is a vital issue after the imaging. It is the precondition of the image analysis. In recent years, the denosing technology make a great progress, some new theories and the technology have been emerged. The Bayesian probability model and multi-resolutions analysis is the emphasis of the research. Some researchers focus on the probability model to find more efficiency and more accurate model. The wavelets methods map the signal to the wavelet domain and exploit the different character of the noise and the signal in wavelet domain with different scale to distinguish the noise and signal. To impose the noise iamge, the mail approach includes the Modulus Maxima reconstruction denosing, spatial denoising, wavelet domain threshold denosing. The tradeoff between the feature keeping and the smoothing still are the main difficulty of the wavelet methods..

Recent studies have shown that, in wavelet sub-band, the statistical model of the image have not in according with the generally Gaussian noise model in time domain. The wavelet coefficients hold a highly kurtotic behaviour in marginal histogram. In literature[1][5][6], the Gaussian Scale Mixtures(GSM) has been proposed. And in [7], the Spatial Varity Gaussian Scale Mixtures (SVGSM) has been proposed as a improvement of the GSM, eg, Mixture Gaussian Scale Mixtures[8], Mixture Projection Gaussian Scale Mixtures(MPGSM) [9]. The model mentioned above reveal explicitly a fundamental non-Gaussian noise model of the nature image of the wavelet coefficients. In many situations, the generally Gaussian Distribution has been used widely as a prior of the wavelet domain. GCD is a symmetric distribution. The main special case is the common Gaussian Distribution and the Laplace distribution. The limit form is $\delta$ function and the Uniformity Distribution. Compare with the Gaussian Distribution, GCD has more complicate study objection and intensive application.

The non local means(NLM) denosing methods have been proved as an efficient denoise method. The framework of the NLM based on the weighted means of the pixels which exploit the high redundancy of the image. The mean algorithm use all the pixels in the image, haven been shown the better results to the Gaussian additive noise. The denoised images have better vision effect and the better evaluation performance in data analysis.

In this paper, firstly, we introduce the steerable pyramid which has the orientation filter property. In the sub-band of the steeralbe pyramid coefficiency, we use the GCD to represent the stasitical characteristic of the image. Based on the modeling of GCD, we proposed a novelty denoise modeling in the wavelet domain. 
The study of the generalized Gaussian distribution and the papameter evaluation of the steerable pyramid.

\section{Nonlocal means ( NLM ) denoising algorithm}

Let image represent by $I$, define $I$ neighborhood $N=\left\{n_{i}\right\}_{i \in I}$, satisfy :

$$
\text { (1) } i \in N_{i} \text {, (2) } i \in N_{i}, j \in N_{i} \Rightarrow i \in N_{j} \text {. }
$$

Then and filtered point $i$, its value $N L M(I(i))$ calculated as a weighted average of all the pixels in the image $I$ follow the equation as

$$
N L M(I(i))=\sum_{j \in I} w(i, j) I(j)
$$

Where the $w(i, j)$ is the weights calculate from the similarity between the pixel $i, j$. And it also holds $\sum_{j \in I} w(i, j)=1,0<w(i, j)<1$.

In most case, $w(i, j)$ calculate as follow:

$$
w(i, j)=\exp \left(-\frac{\left\|E_{i}-E_{j}\right\|_{2, \alpha}^{2}}{h^{2}}\right)
$$

NL-means compute distances between the intensities neighborhoods $E_{i}, E_{j}$ for all the voxels $j$ contained in the certain windows. Based on the original approach, we denote $S$ is the length of the search window, $\mathrm{C}$ is the compare window, and it goes $S>C$. In generally, the size of a noised image is $N^{2}$. So we have to calculate the weight of NL-means with the complexity $N^{2} \times S^{2} \times C^{2}$.

\section{generalized Gaussian distribution denstity function}

Firstly, let

$$
p(x)=\frac{\alpha}{2 \beta \Gamma(1 / \alpha)} \exp \left(-\left|\frac{x}{\beta}\right|^{\alpha}\right)
$$

Where

$$
\beta=\sigma \sqrt{\frac{\Gamma(1 / \alpha)}{\Gamma(3 / \alpha)}}
$$

$\Gamma(z)=\int_{0}^{\infty} e^{-t} t^{z-1} \mathrm{~d} t$ is Gamma function. $\alpha$ and $\beta$ is the shape parameter and the scale parameter. $\sigma^{2}$ is the variance. If $\alpha=1$, GGD is the general Laplace distribution. If $\alpha=2$, GGD is the general Gaussian distribution. When the variance is constant, the GCD distribution curve is more subdued with the increasing of the $\alpha$.

\section{steerable pyramid}

In [10], the steerable pyramid was proposed as an multi-resolution and multi-orientation image wavelet decompose method. Compare with the traditional orthogonal wavelet decomposition, the steerable pyramid decompose the image to a serial orientation sub-band and a lowpass resident band. The orientation is not down-sample, the residential sub-band will not produce the ring artifact by the low-pass filter. The steerable pyramid is tighter framework, the L2 norm is same to the original image. The main shortcoming of the steerabale pyramid is overcomplete decomposition. The overcomplete coefficiency is $\frac{4}{3} K, K$ is the number of the orientation. In this paper, we apply the steerable pyramid to decompose the image for the GCD prior. 
After the steerable pyramid transform, the image decomposed as the highpass sub-band $H_{0}(-\omega)$, lowpass band $L_{0}(-\omega)$, lowpass band and a serial of orientation sub-band $B_{K}(-\omega)$ in sequence and the lower band $L_{1}(-\omega)$.

The literatures have been proved that the image sub-band coefficiency holds the distribution just as GCD, shown the krutotic behavior. Therefore, the key is to achieve the parameter estimation.

The shape papameter $\alpha$ decide whether the GCD can represent the sparse distribution in the margin of the image. We use a moment estimation method to achieve the $\alpha$. Let $\mathrm{x} 1, \mathrm{x} 2 \ldots, \mathrm{xN}$ is the sapmle of the population $\mathrm{X}$. we define the absolute value moment :

$$
m_{1} \quad E\{|\boldsymbol{X}|\}=\sigma \frac{\Gamma(2 / \alpha)}{\sqrt{\Gamma(1 / \alpha) \Gamma(3 / \alpha)}}
$$

Two order moment about the origin is :

Define the function:

$$
m_{2} E\left\{X^{2}\right\}=\sigma^{2}
$$

$$
R(\alpha) \quad \frac{m_{1}^{2}}{m_{2}}=\frac{\Gamma^{2}(2 / \alpha)}{\Gamma(1 / \alpha) \Gamma(3 / \alpha)}
$$

The inverse function can be approximate by hyperbolic function[12] :

$$
R^{-1}(\alpha) \approx \frac{0.2718}{0.7697-\alpha}-0.1247
$$

And the moment $\mathrm{m} 1, \mathrm{~m} 2$ can be approximateas:

$$
\hat{m}_{1}=\frac{1}{N} \sum_{i}\left|x_{i}\right| \quad \hat{m}_{2}=\frac{1}{N} \sum_{i} x_{i}^{2}
$$

therefore, the shape parameter ${ }^{\alpha}$ can be approximate as :

$$
\alpha=R^{-1}\left(\frac{\hat{m}_{1}^{2}}{\hat{m}_{2}}\right)
$$

Scale parameter $\beta$ will be approximate as :

$$
\beta=\sqrt{\hat{m}_{2} \frac{\Gamma(1 / \alpha)}{\Gamma(3 / \alpha)}}
$$

\section{the algorithm of the NLM based on the steerable pyramid}

NLM due with the noise in spatial, its also can impose the image in wavelet domain by the subband of the wavelets transform.

Consider the GCD non local means algorithm, the weight formulation can be:

$$
w(i, j)=\frac{1}{C} \exp \left(-\frac{\left\|E_{i}-E_{j}\right\|_{2}^{\beta}}{(h \alpha)^{\beta}}\right)
$$

The parameter $\alpha$ and $\beta$ coupling together, it make $h$ a little difficult to defines. So we try to decouple the $\alpha$ and $\beta$ make it more simple. So we alter the formulation as:

$$
w(i, j)= \begin{cases}\frac{1}{C} \exp \left(-\left(\frac{\left\|E_{i}-E_{j}\right\|_{2}^{2}}{h^{2}}\right)^{\beta / 2} \frac{1}{\alpha}\right), & \text { if }\left\|E_{i}-E_{j}\right\|_{2}^{2}<h \\ 0, & \text { others }\end{cases}
$$

the improved NLM algorithm is :

$$
N L M(I(i))=\sum_{j \in I} w(i, j) \%(\rho j)
$$




\section{The result and the discussion}

To test the algorithm, we choose other two denosing methods based on the wavelet transform. And we also use two classical spatial domain denoise method Local means filter and median filter. In experiment, firstly, we use $512 \times 512$ as the test image to computer the peak signal-to-noise ratio(PSNR). In NLM, the searcher window is $11 \times 11$, compare window is $7 \times 7 . h^{2}$ is $10 \sigma$ 。 As to the steerable pyramid transform , scale $\mathrm{J}=1$, orientation $\mathrm{K}=4$, we have 4 highpass resident sub-band and a lowepass sub-band. We can see from the figure 3 the proposed methods have better vision effect. According table 1, the proposed method shown better PSNR

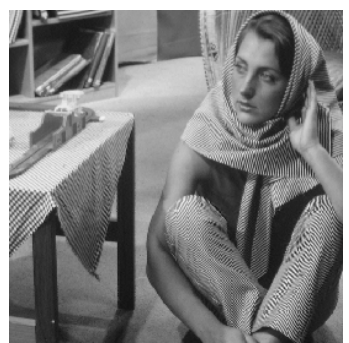

(a) original image

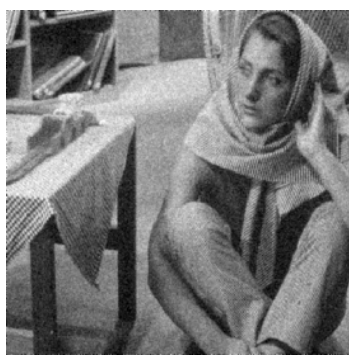

( e ) LM filter

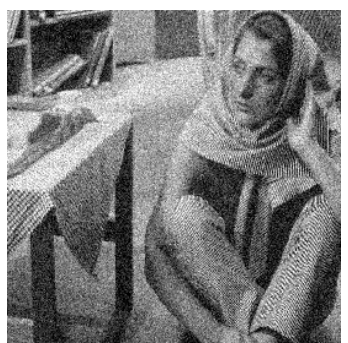

(b) noisy iamge $\sigma=0.01$

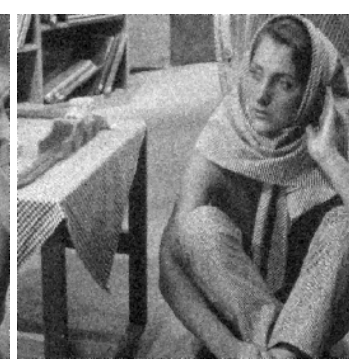

( f ) Mid filter

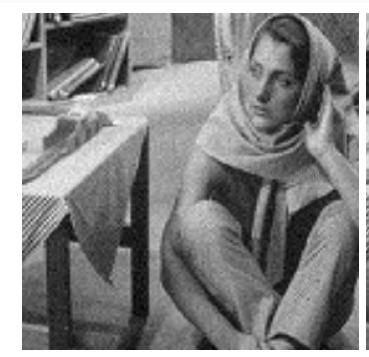

(c) hard threshold

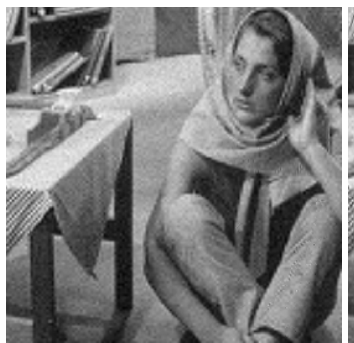

(g)NLM

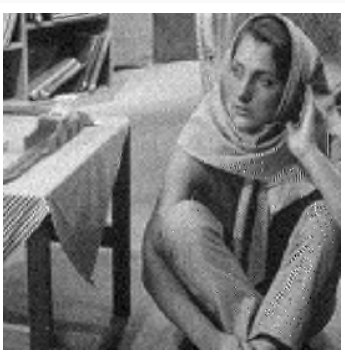

(d) soft thereshold

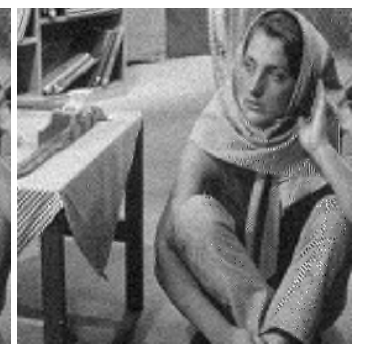

(h)Proposed algorithm

\begin{tabular}{llll}
\multicolumn{1}{r}{ TABLE I. } & \multicolumn{2}{c}{ TABLE THE PSNR OF SOME IMAGE DENOISNG } \\
\hline & PSNR & \\
\cline { 2 - 4 } Algorithm & $\sigma=10$ & $\sigma=10$ & $\sigma=10$ \\
& 71.4 & 69.5 & 62.6 \\
$\begin{array}{l}\text { hard } \\
\text { threshold } \\
\text { Soft } \\
\text { thereshold }\end{array}$ & 71.6 & 69.5 & 62.6 \\
LM fitler & 72.1 & 68.7 & 60.2 \\
MID filter & 73 & 67.4 & 58.6 \\
& 73.2 & 70.1 & 63.3 \\
Typical NLM & 73.2 & 70.5 & 63.9 \\
Our algorithm & & &
\end{tabular}

\section{Conclusion}

In this paper, we have been proposed an efficient denosing methods based on the steerable pyramid wavelet transform. In the domain, the coefficient exposed the GCD characteristic which is indifferent with the traditional Gaussian distribution, so we improve the NLM method to deal the GCD model in wavelet domain. The results shown that the proposed methods have better denoise effect. The follow-up work focus on the optimal of the NLM approach and the better approximation method for the GCD parameter. 


\section{Acknowledgment}

The work is supported by the Fund Project of Southwest University for Nationalities of China (12NZYTD22).

\section{References}

[1] D. Andrews and C. Mallows, "Scale mixtures of normal distributions", J. R. Statist. Soc, vol. 36, pp.99 1974

[2] D. L. Donoho and I. M. Johnstone, "Ideal spatial adaptation by wavelet shrinkage", Biometrika, vol. 81, no. 3, pp.425 -455 1994

[3] F. Abramovich, T. Sapatinas, and B. W. Silverman, "Wavelet thresholding via a Bayesian approach", J. R. Statist. Soc. B, vol. 60, pp.725 -749 1998

[4] E. P. Simoncelli, P. M\\&uuml;ller and B. Vidakovic, "Bayesian denoising of visual images in the wavelet domain", Bayesian Inference in Wavelet Based Models, vol. 141, pp.291 -308 1999 :Springer-Verlag

[5] J. Portilla, V. Strela, M. Wainwright, and E. Simoncelli, Image Denoising Using Gaussian Scale Mixtures in the Wavelet Domain, 2002 :Courant Inst. of Math. Sci., New York University

[6] J. Portilla, V. Strela, M. J. Wainwright, and E. P. Simoncelli, "Image denoising using scale mixtures of Gaussians in the wavelet domain,” IEEE Trans. Image Proc., vol. 12, pp. 1338-1351, November 2003.

[7] J. A. Guerrero-Col ' on, L. Mancera, and J. Portilla, "Image restoration using space-variant gaussian scale mixture in over-complete pyramids," IEEE Trans. Image Proc., vol. 17, no. 1,pp. 27 - 41, January 2008.

[8] D. K. Hammond and E. P. Simoncelli, "Image denoising with an orientation-adaptive Gaussian scale mixture model," in IEEE Int'l Conf. on Image Proc., Atlanta, GA, October 2006.

[9] J. Portilla and E. P. Simoncelli, "A parametric texture model based on joint statistics of complex wavelet coefficients", Int. J. Comput. Vis., vol. 40, no. 1, pp.49 -71 2000

[10] Wan, T. Canagarajah, N. Achim, A. "Segmentation of noisy colour images using Cauchy distribution in the complex wavelet domain", IET Image Processing, Vol.5, Iss.2, pp.159, 2011, ISSN: 17519659http://dx.doi.org/10.1049/iet-ipr.2009.0300

[11] Wong, Alexander Mishra, Akshaya Zhang, Wen Fieguth, Paul Clausi, David A. "Stochastic image denoising based on Markov-chain Monte Carlo sampling", Signal Processing, Vol.91, Iss.8, pp.2112, 2011, ISSN: 01651684 